\title{
Evidence of a role for Th17 cells in the breach of immune tolerance in arthritis
}

\author{
Xinhua Yu ${ }^{1,2}$ and Saleh M Ibrahim*3
}

\begin{abstract}
Th17 cells are thought to play a pathogenic role in various autoimmune diseases. Cytokines secreted by Th17 cells like IL-17, IL-17F and IL-22 have the capacity to mediate a massive inflammatory response. These proinflammatroy cytokines are likely to mediate the pathogenic potential of Th17 cells. Recent evidence suggests a role for Th17 cells in the breach of immune tolerance. This might shed some new light on the pathogenic role of Th17 cells in autoimmunity.
\end{abstract}

In this issue, $\mathrm{Yu}$ and colleagues [1] present a report on gene expression profiles of antigen (Bhsp65)-tolerized and preclinical arthritic rats with adjuvant-induced arthritis (AIA). AIA is a widely used animal model of rheumatoid arthritis, which is inducible in rats by immunization with Mycobacterium tuberculosis (Mtb) in complete Freund's adjuvant [2]. It is a predominately CD4 $\mathrm{T}$ cell-mediated chronic disease with clearly identifiable disease phases, including incubation, onset, peak, and recovery [3,4]. AIA has many features resembling rheumatoid arthritis; therefore, it has been extensively used for investigating the pathogenesis of rheumatoid arthritis and in preclinical studies. The Mycobacterial $65 \mathrm{kDa}$ shock protein (Bhap65) has been identified as an important disease-related antigen in AIA [4]. Pretreatment of rats with Bhsp65 protect them from AIA, providing a good model for investigating immune tolerance $[4,5]$.

In a first step, $\mathrm{Yu}$ and colleagues investigated the gene expression profiles of lymph node (LN) cells of arthritic rats at different clinical phases of AIA. Interestingly, compared with naive controls, maximal gene expression changes were observed in LN cells of rats in the incubation phase instead of the peak or recovery phases. Most differentially expressed genes at the incubation

*Correspondence: saleh.ibrahim@uk-sh.de

3Department of Dermatology, University of Lübeck, 23538, Lübeck, Germany

Full list of author information is available at the end of the article phase were upregulated, while most at the peak and recovery phases were downregulated. This observation is consistent with findings from a collagen-induced arthritis study in mouse [6], suggesting that immunological processes are active in the LN during this preclinical stage and that this process might be critical for arthritis development.

Aiming to identify the gene expression signature of Bhsp65-induced tolerance, $\mathrm{Yu}$ and colleagues further investigated the gene expression profiles of Bhsp65tolerized and preclinical arthritic rats. They observed only minor differences in the gene expression profiles of LN cells in the incubation phase between Bhsp65tolerized and preclinical arthritic rats. However, when they stimulated those cells with Bhsp65 in vitro, dramatic differences were observed between cells from Bhsp65tolerized and preclinical arthritic rats compared with unstimulated controls. By comparing the gene expression profiles of Bhsp65-tolerized rats and preclinical arthritic rats, 12 genes were identified as encoding signature molecules of the Bhsp65-induced tolerance, including Cd86, Il1b, Lta, Socs3, Il10, Vegfa, Ifi27l1, Il17a, Il17f, Il22, Il33 and Cxcr7. Interestingly, 5 of those 12 signature molecules are Th17 cell related. Ill7a, Il17f, and Il22 encode the signature cytokines of Th17 cells [7], while proteins encoded by Socs 3 and $I l 10$ are essential negative regulators of Th17 cells $[8,9]$.

Since $I l 17 a, I l 17 f$ and $I l 22$ were upregulated in only the preclinical arthritic rats while Socs 3 and $I l 10$ were upregulated in only the Bhsp65-tolerized rats, it shows that there were strong Th17 responses to in vitro stimulation with Bhsp65 in the preclinical arthritic group. The Th17 response to Bhsp65 is absent in the Bhsp65-tolerized group, and this absence might be due to the suppression of Th17 negative regulators (Figure 1). In contrast to Th17 cell-related genes, the Th1 and Th2 cell-related genes Ifng and Il4 behave differently, that is, Ifng is upregulated and Il4 is unchanged in both tolerized and preclinical arthritic groups. These results suggests that Th17 cells might play a role in the breach of immune tolerance.

Th17 cells are a recently identified subset of CD4 $\mathrm{T}$ cells, with a primary function of clearing extracellular pathogens during infections [7]. Although they have been recognized as key players in adaptive immunity, they 


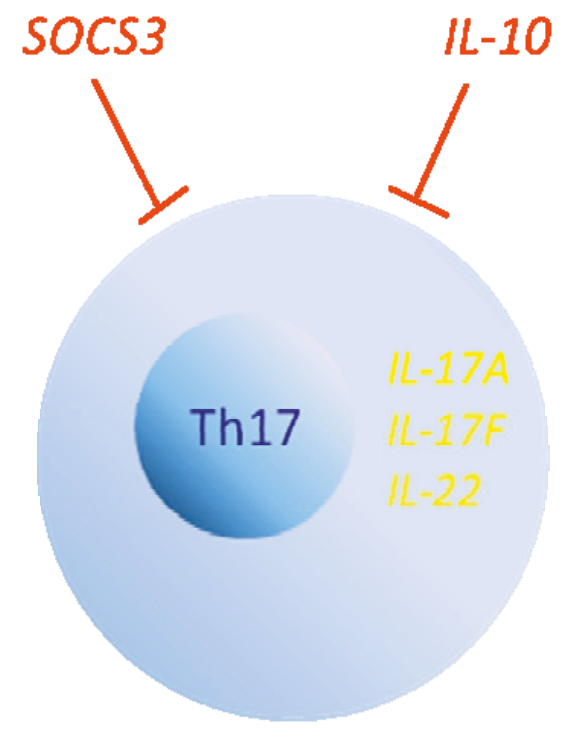

Figure 1. Five Th17-related signature genes of Bhsp65-induced tolerance in adjuvant-induced arthritis. Upon stimulation with Bhsp65 on lymph node cells, genes indicated in yellow are upregulated in preclinical arthritic rats but not changed in Bhsp65-tolerized rats, while genes indicated in red are upregulated in Bhsp65-tolerized rats but not preclinical arthritic rats.

were initially identified in autoimmune disease research in $2005[10,11]$. Since then, extensive studies have been performed and confirmed a pathogenic role of Th17 cells in various autoimmune diseases and their animal models $[7,12]$. Nevertheless, the pathogenic characteristics of Th17 cells in autoimmunity are only partially known. Studies have already shown that Th17 cells secrete cytokines, including IL-17, IL-17F and IL-22, and are able to mediate strong inflammatory responses [7]. These proinflammatory cytokines could explain, at least partially, the pathogenic characteristics of Th17 cells in autoimmunity. However, whether other components contribute to the pathogenic characteristics of Th17 cells is not clear.

Recently, studies have investigated the role of Th17 cells in immune tolerance. In 2008, Wang and colleagues [13] reported that IL-17 could interfere with MBP 68-86-induced immune tolerance in experimental autoimmune encephalomyelitis. In 2009, Satpute and colleagues [14] reported that Bhsp65-induced tolerance in AIA is associated with the reduction of IL-17 production. More recently, Peron and colleagues [15] showed that oral tolerance is associated with Th17 cell reduction in experimental autoimmune encephalomyelitis. Taken together, these studies suggest that Th17 cells might play a role in the breach of immune tolerance, pointing to a new mechanism mediating their pathogenic role in autoimmunity. However, this needs to be validated and the mechanism behind it needs to be investigated further.

\section{Abbreviations}

AIA, adjuvant-induced arthritis; IL, interleukin; LN, lymph node; Mtb, Mycobacterium tuberculosis.

\section{Author details}

'Laboratory of Autoimmunity, The Medical College of Xiamen University, Xiamen University, 361005, China. ${ }^{2}$ Section of Immunoregulation, Research center Borstel, Borstel, 23845, Germany. 3Department of Dermatology, University of Lübeck, Lübeck, 23538, Germany.

\section{Published: 31 October 2011}

\section{References}

1. Yu H, Lu C, Tan M, Moudgil K: The gene expression profile of preclinical autoimmune arthritis and its modulation by a tolerogenic diseaseprotective antigenic challenge. Arthritis Res Ther 2011, 13:R143.

2. PEARSON CM: Development of arthritis, periarthritis and periostitis in rats given adjuvants. Proc Soc Exp Biol Med 1956, 91:95-101.

3. Pearson CM, Waksman BH, Sharp JT: Studies of arthritis and other lesions induced in rats by injection of mycobacterial adjuvant. V. Changes affecting the skin and mucous membranes. Comparison of the experimental process with human disease. J Exp Med 1961, 113:485-510.

4. van Eden W, Thole JE, van der Zee R, Noordzij A, van Embden JD, Hensen EJ, Cohen IR: Cloning of the mycobacterial epitope recognized by T lymphocytes in adjuvant arthritis. Nature 1988, 331:171-173.

5. Moudgil KD, Chang TT, Eradat H, Chen AM, Gupta RS, Brahn E, Sercarz EE: Diversification of $\mathrm{T}$ cell responses to carboxy-terminal determinants within the $65-\mathrm{kD}$ heat-shock protein is involved in regulation of autoimmune arthritis. J Exp Med 1997, 185:1307-1316.

6. Yu X, Bauer K, Koczan D, Thiesen HJ, Ibrahim SM: Combining global genome and transcriptome approaches to identify the candidate genes of smalleffect quantitative trait loci in collagen-induced arthritis. Arthritis Res Ther 2007, 9:R3

7. Korn T, Bettelli E, Oukka M, Kuchroo VK: IL-17 and Th17 cells. Annu Rev Immunol 2009, 27:485-517.

8. Chen Z, Laurence A, Kanno Y, Pacher-Zavisin M, Zhu BM, Tato C, Yoshimura A, Hennighausen L, O'Shea JJ: Selective regulatory function of Socs 3 in the formation of IL-17-secreting T cells. Proc Natl Acad Sci U S A 2006 103:8137-8142.

9. Huber S, Gagliani N, Espluques E, O'Connor W Jr, Huber FJ, Chaudhry A, Kamanaka M, Kobayashi Y, Booth CJ, Rudensky AY, Roncarolo MG, Battaglia M, Flavell RA: Th17 cells express interleukin-10 receptor and are controlled by Foxp3 and Foxp3+ regulatory CD4+ T cells in an interleukin-10-dependent manner. Immunity 2011, 34:554-565.

10. Cua DJ, Sherlock J, Chen Y, Murphy CA, Joyce B, Seymour B, Lucian L, To W, Kwan S, Churakova T, Zurawski S, Wiekowski M, Lira SA, Gorman D, Kastelein RA, Sedgwick JD: Interleukin-23 rather than interleukin-12 is the critical cytokine for autoimmune inflammation of the brain. Nature 2003, 421:744-748.

11. Langrish CL, Chen Y, Blumenschein WM, Mattson J, Basham B, Sedgwick JD, McClanahan T, Kastelein RA, Cua DJ: IL-23 drives a pathogenic T cell population that induces autoimmune inflammation. J Exp Med 2005, 201:233-240.

12. Oukka M: Th17 cells in immunity and autoimmunity. Ann Rheum Dis 2008, 67 Suppl 3:iii26-iii29.

13. Wang GY, Sun B, Kong QF, Zhang Y, Li R, Wang JH, Wang DD, Lv GX, Li HL: IL-17 eliminates the therapeutic effects of myelin basic protein-induced nasal tolerance in experimental autoimmune encephalomyelitis by activating IL-6. Scand J Immuno/ 2008, 68:589-597.

14. Satpute SR, Rajaiah R, Polumuri SK, Moudgil KD: Tolerization with Hsp65 induces protection against adjuvant-induced arthritis by modulating the antigen-directed interferon-gamma, interleukin-17, and antibody responses. Arthritis Rheum 2009, 60:103-113.

15. Peron JP, Yang K, Chen ML, Brandao WN, Basso AS, Commodaro AG, Weiner $\mathrm{HL}$, Rizzo LV: Oral tolerance reduces Th17 cells as well as the overall inflammation in the central nervous system of EAE mice. J Neuroimmunol 2010, 227:10-17.

doi:10.1186/ar3490

Cite this article as: Yu X, Ibrahim SM: Evidence of a role for Th17 cells in the breach of immune tolerance in arthritis. Arthritis Research \& Therapy 2011, 13:132. 